\section{DESIGN OF TEST RIGS FOR MEASURING DYNAMIC PROPERTIES OF SOFT ACTUATORS}

MONIKA TROJANOVA, ALEXANDER HOSOVSKY

Technical University of Kosice, Faculty of Manufacturing Technologies with the Seat in Presov, Slovakia

DOI: 10.17973/MMSJ.2020_03_2020005

monika.trojanova@tuke.sk

The paper describes the process of design and construction of test rigs, whose task will be to measure dynamic characteristics (namely displacement, pressure, force). The test rigs are built for three specific types of soft drives - fluid muscle, DE actuator (dielectric elastomer) and IMPC actuator (ionic polymer metal composite). The measuring devices, which have been assembled at the authors' workplace, will serve to study the displacement in soft drives with the supposed synergistic effect achieved by a comparative approach. These drives exhibit complicated behaviour in terms of displacement control. The test rigs will measure also other non-linear characteristics in the future.

KEYWORDS

Soft Actuators, Fluid Muscle, Dielectric Elastomer Actuator (DE), Ionic Polymer Metal Composites Actuator (IPMC), Nonlinear Dynamic Characteristic

\section{INTRODUCTION}

The current trend in robotics is also focused on research and development of soft drives, which should support and move the automation process to a higher level while ensuring safe cooperation with humans. The demands on it are currently much higher, because we meet in practice with the requirements of high adaptation and flexibility. At the same time, it is the work, both in demanding operating conditions and in work areas, where the working zones of robots and employees interact. The solution could be highly flexible handling equipment, soft robots or collaborative robots (cobots). These soft robots can increase the level of automation, ensure a safe interaction with a person, and at the same time "free" a person from manual work, while the employee's potential can be used for more creative activities with a higher degree of flexibility. Soft robots are of different materials and have different types of drives (soft drives) than conventional types of robots. This means that is also needed change in approach when examining the basic properties and characteristics of soft drives. [KEEPING 2018], [MISITI 2018]

There are several publications in the scientific and research spheres that are focused on the problem of soft drives. For example, in [OKUZAKI 2014], there is a broad overview of contributions in this area. The publication is divided into several sections focusing on soft drive materials, soft drive modeling, application and future of bioactuators. They also describe two studied soft drives (DE actuator and principle of its operation in [CHIBA 2014] as well as IPMC actuator in [KIKUCHI 2014]). A similar review is provided by Electroactive Polymers for Robotic Applications (edited by K.J. Kim and S. Tadokoro), which is oriented on electroactive polymers for use in robotics. E.g. in [SAMATHAM 2007] is an overview of active polymers such as non-electroactive and electroactive polymers. Non-electroactive polymers include e.g. chemically active polymers, shape memory polymers and others. Electroactive polymers include ferroelectric polymers (where there is also a DE actuator) and ionic polymer gels (including IPMC). Pneumatic artificial muscles (PAM) are the subject of research by many known researchers. For example, Wickramatunge in [WICKRAMATUNGE 2010] describes the mechanical properties of PAM. The authors of the paper also have experience with this type of soft drive. Their area is the investigation of PAM hysteresis e.g. in [TROJANOVÁ 2017], but also identification the dynamics of experimental manipulators that are driven by Festo fluid muscles [TROJANOVÁ 2018], [HOŠOVSKÝ 2016]

The content of the article is focused on the design and construction of two test rigs, which are intended to measure the dynamic characteristics of three types of soft actuators in the future and thus support research in the field of soft drives. Specifically, it will be the fluid muscle, DE actuator and IPMC actuator, whose principle of operation is explained in the following part of the article. Subsequently, the chapter follows, where the construction of the test rigs is presented by real images, and at the same time there is a list of used components and devices that were necessary for the assembly of such test rigs. The last part of the paper explains and graphically presents the principle of measure one of the characteristics- displacement (bending) of all three types of actuators, which will be of interest and research soon.

\section{SOFT ACTUATORS AND PRINCIPLE OF THEIR FUNCTIONS}

Most soft actuators were discovered based on the monitoring of movements of living organisms that respond to stimulus such as heat, humidity, magnetic field, electric field and others. Soft drives also use a similar principle of changing shape or volume based on an external stimulus. These actuators can convert physical or chemical energy into mechanical work, and their movement is more flexible than conventional drives (e.g. electric motors). They are predominantly made of natural or synthetic materials such as biomaterial, elastomer, gel and others. Historically, the first soft drives were gel-based actuators that responded to $\mathrm{pH}$ changes (the half of the 20th century). In the second half of the 20th century, other actuators were made of the same material, but they were also able to react to stimulus such as salt, light, magnetic and electric fields. With the arrival of the new millennium, other materials began to be used. [OKUZAKI 2014]

The authors chose dielectric elastomer (DE), fluid muscle and ion polymer metal composite (IPMC) for the research of soft drives. The material from which they are made, as well as the principle of their operation, is described in the following section.

\subsection{The fluid muscle FESTO}

Fluid muscle Festo belongs to the category of pneumatic artificial muscles, which are one of the best known drives in the field of soft robotics. However, the properties of the described muscle are better than the basic type of pneumatic artificial muscles (such as McKibben's artificial muscle). Thanks to the unified outer and inner layers in one, it eliminates friction and has a better force-to-weight ratio. Next advantage is, that it has a relatively low weight and low maintenance. The disadvantages as a hysteresis or non-linearity of characteristics complicating the identification and control process. The muscle, as can be 
seen in Fig. 1, consists of two basic parts: an inner tube and an outer braid, and endings. The working medium of this type of drive is compressed air. The principle of muscle function is simple. After the air is let into the muscle by a compressor, in the muscle created contractions (i.e. the muscle is shortened, its diameter also increases). This contraction ultimately causes an external tensile force. [FESTO 2017], [TROJANOVÁ 2019]

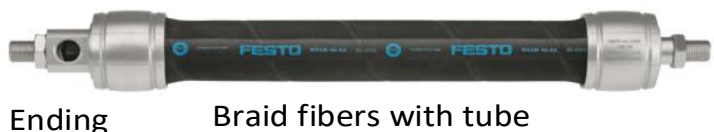

Figure 1: Fluid muscle from manufacturer FESTO [FESTO 2017]

\subsection{DE actuator}

The dielectric elastomer is a type of actuator that consists of an elastomeric layer sandwiched between two thin electrodes, as can be seen in Fig. 2. Principle of operation the DE actuator is follows: the elastomer is a non-conductive material and when the electrodes are connected to a high-power source, the electrodes are attracted to each other (since one is negative, the other positive). The reaction of the elastomer to the resulting electrostatic field is deductible, the elastomer is thinned and elongated in the other two possible directions. Since the elastomer is flexible, the electrodes and the elastomer return to its original position when the electrostatic field is lost. Normally, the thickness of the elastomer is in the range of 0.1-0.3 mm, but there are also elastomers with a much smaller thickness. The material of which the elastomeric layer is made can be silicone or polyurethane. The electrodes must also meet the standard of certain flexibility. A metal electrode as thin sheet hasn't good properties for deformation of DE actuator and therefore conductive lubricants (silicon containing carbon or silver) are used. [OKUZAKI 2014]

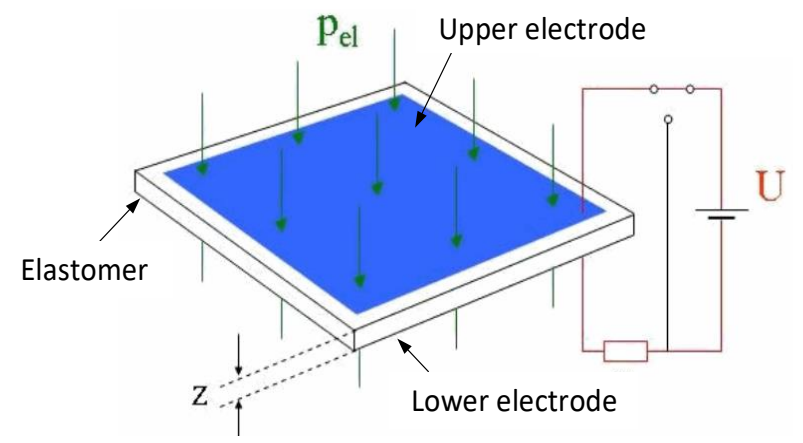

Figure 2: DE actuator [CHIBA 2014]

\subsection{IPMC actuator}

In Fig. 3 is a diagram of the operation of an IPMC actuator whose abbreviation means an ionic polymer metal composite. This actuator consists of two electrodes made of noble metal (e.g. platinum), and between them is a conductive ion-polymer gel membrane. In the figure, the red dots indicate the free cations present in the membrane and the blue dots are water molecules. When connected the electrodes to a power source, free cations and water molecules attract to a negatively charged electrode, causing the IPMC to bend towards the anode. The advantages of such drives are low voltage, high flexibility, high degree of deformation. The disadvantage is the constant presence of water molecules, but this is not so much an obstacle if the device in a humid environment. [KIKUCHI 2014]

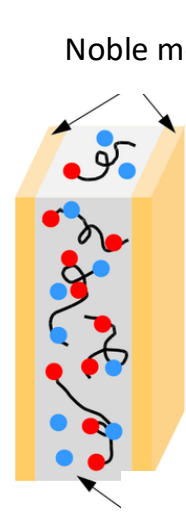

After applying voltage
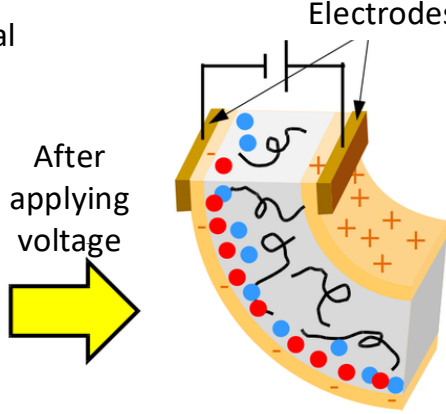

Ionic-polymer membrane

\begin{tabular}{|cc|}
\hline - & Free cations \\
en & Molecules of water \\
Connections
\end{tabular}

Figure 3: IPMC actuator [OKUZAKI 2014]

\section{CONSTRUCTION OF TEST RIGS}

Overall, two test rigs were designed and constructed to measure especially the static and dynamic hysteresis of soft actuators. First from them will be used to measure the characteristics of artificial muscle DMSP-5, while by design of the test rig was coming out from previous experience gained during researches of fluid muscles. Of course, it was also necessary to think of the contractile properties of the muscle (shortening, diameter increase) as well as the source of the working medium compressed air by a compressor. The second assembled test rig will serve to measure the characteristics of DE and IPMC actuators. The design of this experimental preparation was based primarily on the properties of the actuators themselves and on the differences compared to artificial muscles, such as e.g. unlike the artificial muscle, which becomes shorter in contraction, the DE actuator is extended; The DE actuator needs a relatively high power source for its correct operation; The IPMC actuator, as it works on the basis of free water molecules, must not dry out and others.

\subsection{Test rig for measuring the characteristic of IPMC and DE actuators}

Fig. 4 shows an implemented test rig needed to be measurement the dynamic characteristics of two types of actuators (namely, displacement of DE actuator and bending of IPMC actuator). The main part of the structure is made from aluminium profiles, which allow for possible modification of the product, its compactness and its transfer.

The following components and devices were used to construction of the test rig for sensing of displacement DE actuator:

- DE actuator,

- Panasonic HL-G105-A-C5 Optical Laser Sensor (1.5 $\mu \mathrm{m}$ resolution, $\pm 10 \mathrm{~mm}$ range),

- PC with DAQ card MF 624 HUMUSOFT,

- Corad KA 3005 D-3S power source (0-30 V, 0-5A),

- HV generator (0-2500 V), 
- a precise weight with nominal value of $50 \mathrm{~g}$ ( $\pm 3 \mathrm{mg}$ tolerance),

- a precise weight with nominal value of $100 \mathrm{~g}$ ( $\pm 5 \mathrm{mg}$ tolerance).

The following components and devices were used to construction of the test rig for sensing of displacement (bending) IPMC actuator:

- IPMC actuator,

- Panasonic HL-G105-A-C5 Optical Laser Sensor, (1.5 $\mu \mathrm{m}$ resolution, $\pm 10 \mathrm{~mm}$ range),

- PC with DAQ card MF624 HUMUSOFT,

- TDK Lambda programmable DC power source (0-100 V, 0-6 A).

PC with DAQ card

$$
\text { IPMC }
$$
actuator

Precise

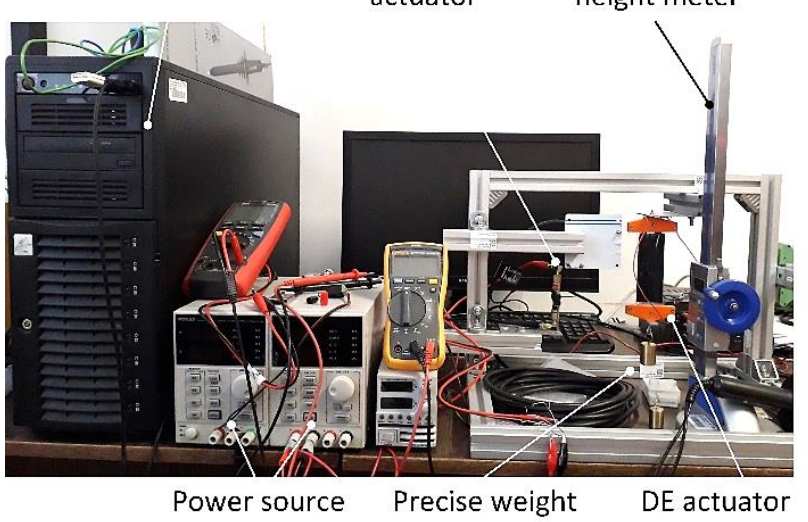
height meter

Figure 4: Test rig for measuring dynamic characteristics of IPMC and DE actuators

\subsection{Test rig for measuring the characteristic of fluid muscle}

Fig. 5 shows test rig, that will serve, inter alia, to measure characteristic of displacement the DMSP-5 fluid muscle from Festo. Like the first test rig, the supporting structure is predominantly made of aluminium profiles.

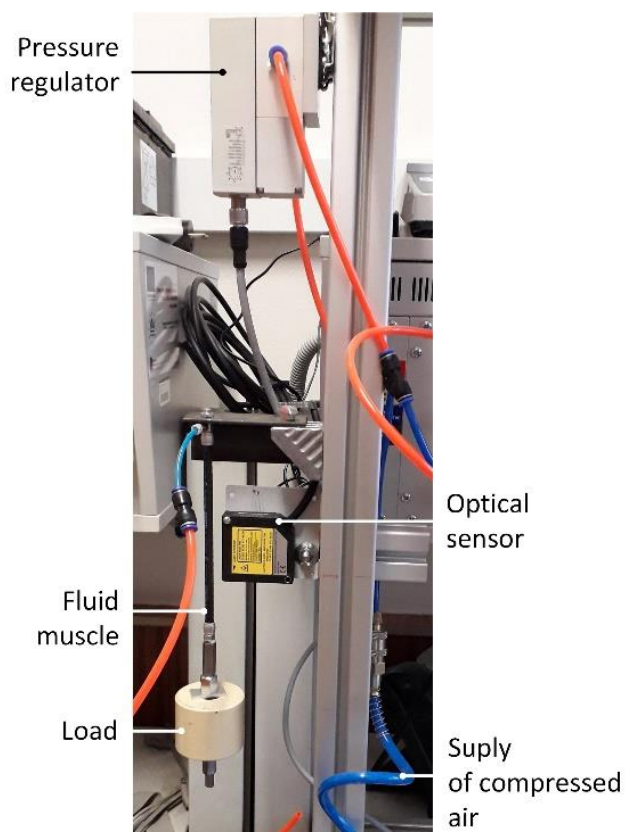

Figure 5: Test rig for measuring dynamic characteristics of DMSP-5 fluid muscle
The following equipment was used to build the experimental test rig:

- Festo fluid muscle DMSP-5 (length $150 \mathrm{~mm}$, diameter $5 \mathrm{~mm}$ ),

- Scheppach HC 53dc compressor (0-1000 kPa pressure),

- proportional pressure regulator Festo VPPM-6L,

- Panasonic HL-G112-A-C5 Optical Laser Sensor ( $8 \mu \mathrm{m}$ resolution, $\pm 60 \mathrm{~mm}$ range),

- PC with DAQ card MF644 HUMUSOFT,

- Corad KA3010D power source (0-30 V, 0-10 A),

- load with weight $1 \mathrm{~kg}$.

\section{PRINCIPLE OF MEASURING THE DISPLACEMENT FOR INDIVIDUAL ACTUATORS}

The principle of displacement measurement, resp. bending for the IPMC actuator is shown in Fig. 6. An IPMC strip is attached between two electrodes on an aluminium profile test rig. When the electrodes are connected to a DC voltage source, bending occurs (provided the strip is wet and thus contains water molecules). This bend is measured from above by an optical laser sensor which records a change in the position of the point with a resolution of $1.5 \mu \mathrm{m}$ within $\pm 10 \mathrm{~mm}$. Since the displacement of the actuator $\Delta$ (or $\delta$ ) varies depending on the free length of the strip I (length from the actuator attachment between the electrodes to the measurement point), so the radius of curvature $R$ can be determined by equation (1). The measurement process will be carried out without loading the actuator. The measured displacement values will be recorded using a DAQ card and processed by computer technology and Matlab software.

$\frac{1}{R}=\frac{2 \delta^{2}}{l^{2}+\delta^{2}}$

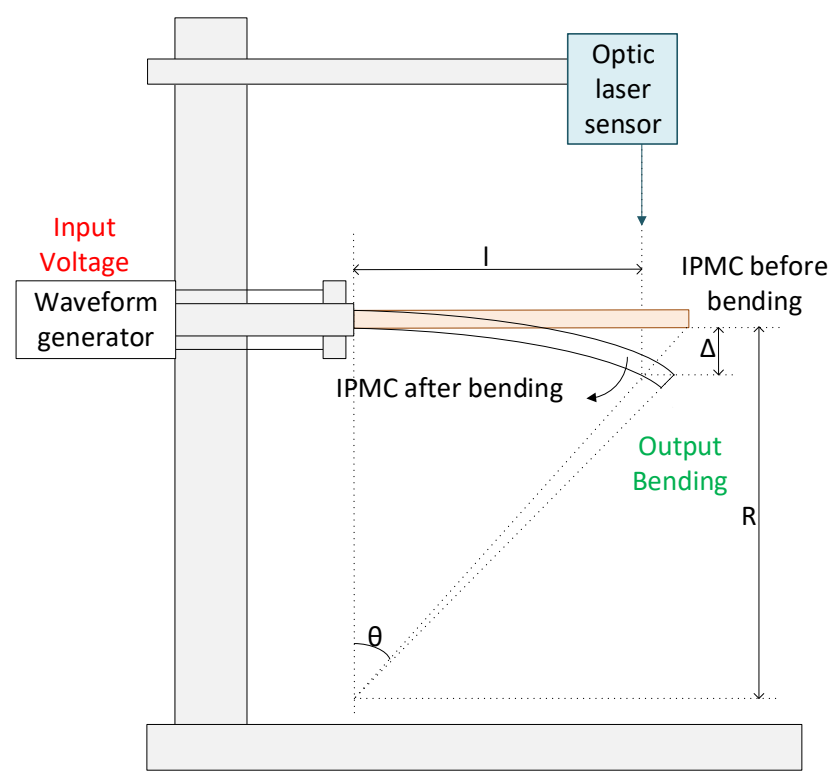

Figure 6: Schematic diagram of IPMC actuator bending measurement

The displacement measurement principle for the dielectric elastomer is shown in Fig. 7. The elastomer itself does not have a large volume change, but when is placed between the electrodes, its volume and shape change is controlled and usable. For correct operation of the DE actuator it is necessary to ensure that the actuator has to be tensioned. This condition is fulfilled for measurement in Fig.7, because the actuator is attached with one ending to the top of the profile of the test rig 
by one end and at the other end is placed a weight with nominal value of $50 \mathrm{~g}$ or $100 \mathrm{~g}$. If is DE actuator connected to a power source and a high-voltage generator, is actuator inter alia extended, which is sensed by an optical laser sensor. The sensor is used the same as for IPMC and therefore its parameters are identical. Similar to sensing displacement in artificial muscle, in $\mathrm{DE}$, the laser jet of the sensor does not fall on the actuatordeformed part of the actuator but on the fixed part of the actuator. The evaluation of the measurements is carried out by means of computer technology and software support Matlab.

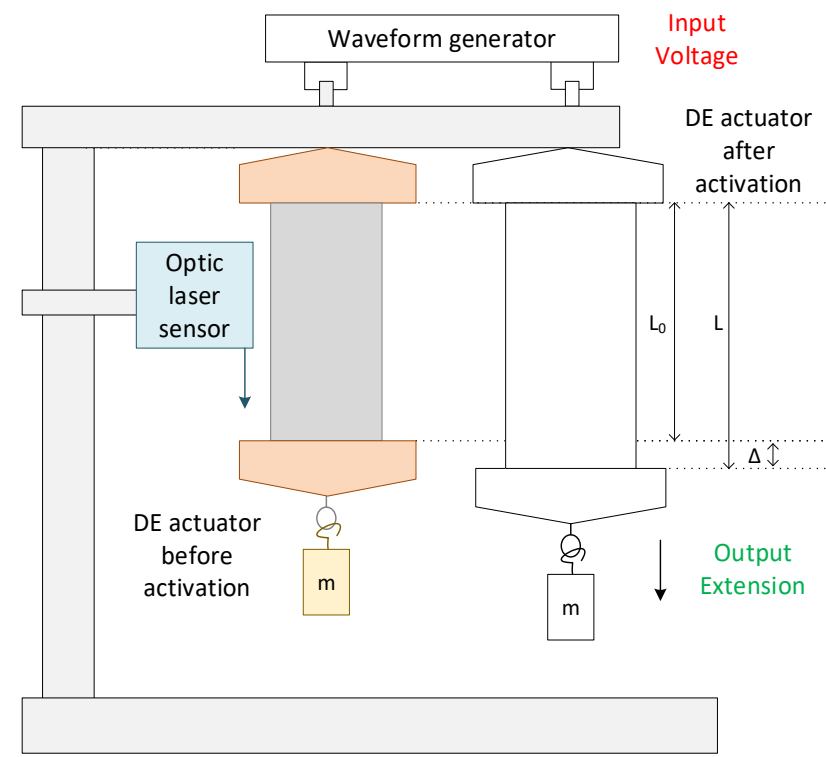

Figure 7: Schematic diagram of DE actuator displacement measurement

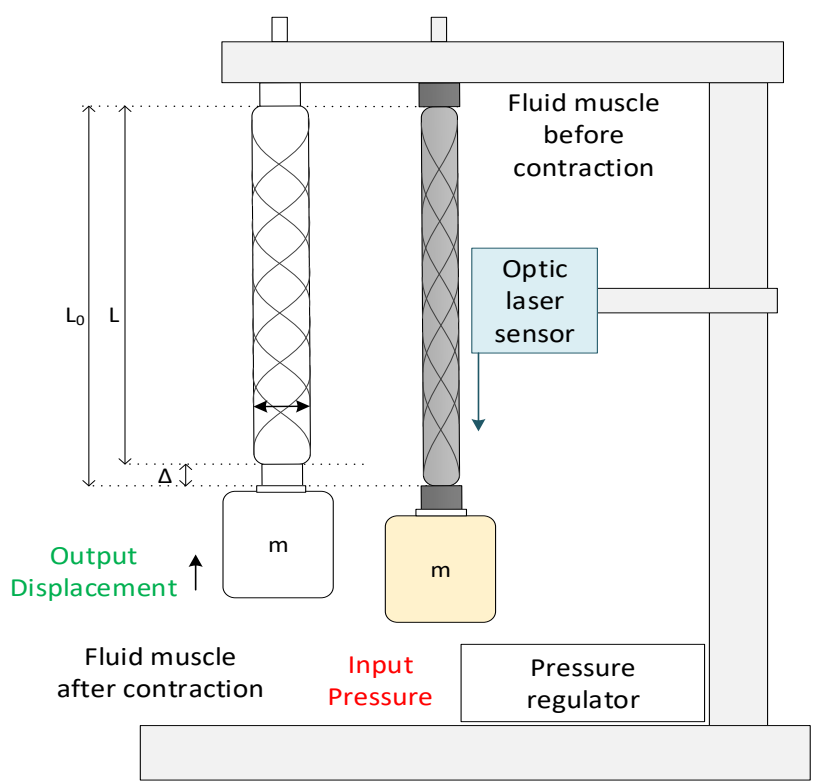

Figure 8: Schematic diagram of DMSP-5 fluid muscle displacement measurement

For sensing of displacement of the fluid muscle DMSP-5 will be apply the following sequence of steps: one end of the muscle is attached to the upper construction of the device by ending. At the other end of the muscle is placed a $1 \mathrm{~kg}$ weight, that ensure a constant load and thus measurement under isotonic conditions. The working medium (compressed air) will be let into the muscle using a compressor. The change of pressure in the tube causes a contraction of muscle, which is mathematically formulated by equation (2), where $L_{0}$ is the initial length of the muscle (muscle is empty) and $L$ is the length of the muscle after contraction.
$\kappa=\left(1-\frac{L}{L_{0}}\right)$

The required muscle pressure is achieved by a proportional pressure regulator from Festo. The optical laser sensor is located on the side of the test rig so the laser jet can detect the shift of weight when the muscle is pull (see Fig. 8). The sensor can sense with a resolution of $8 \mu \mathrm{m}$ and a range of $\pm 60 \mathrm{~mm}$. The measured values of displacement will be recorded using a DAQ card and processed by a computer and Matlab software.

The manipulated variable for a fluidic muscle is control voltage for proportional pressure regulator. If the dynamics of regulator itself is neglected, the pressure within muscle can be considered proportional to the set value of control voltage. The range of input signal for used pressure regulator is $0-10 \mathrm{~V}$, but for the experimentation only $0-6 \mathrm{~V}$ range will be used due to the maximum pressure of DMSP-5 (600 kPa). It is expected that with regard to the measured characteristics of a muscle staircase, triangular and harmonic excitation signals will be used. These types of signals can be easily created in software (e. g. Matlab) and fed to the output of DAQ card. When using proportional pressure regulators, no other electronic components (for signal conditioning) are needed. Similar types of excitation signals will be used both for IPMC and DEA. DEA needs very high voltages (up to $2500 \mathrm{~V}$ ), which need to be provided by HV generator - this can be controlled using a low-level (0-5V) voltage signal directly from DAQ card. Similarly, the control voltage of IPMC will be limited to $0-5 \mathrm{~V}$ (to limit the possible electrolysis) but it cannot be controlled directly from DAQ card. For this purpose, programmable DC power source (TDK-Lambda) can be used.

\section{CONCLUSIONS}

In the paper was introduced the construction of the test rigs, which will allow as a device for measuring the characteristics of three types of soft drives. Currently, test rigs are adapted to measure characteristics at isotonic conditions. Later the test rigs will be adapted for measurement at isometric conditions. The primary aim will be to measure both static and dynamic hysteresis when will be monitoring input/output dependence: for IPMC actuator the dependence of voltage/bending, for DE stress the dependence of voltage/extension, and for muscle the dependence of pressure/displacement. In terms of dynamics, these dependencies will be monitored at different frequencies.

At the same time as the process of designing and assembling the preparations, the phase of summarizing the knowledge base from the areas of fluid muscles and electroactive polymers was also taking place. The next step will be research itself, which will consist of several steps. First, it will need to be performed the measurements to obtain a sufficiently representative set of data for the identification (experimental modeling) process. Subsequently, analysis and application of methods suitable for drive modeling will be performed. Methods will be chosen from the area of computational intelligence - neural networks, fuzzy models, evolutionary computations and others. The last step will be the validation of the identified models on the test data set, which will be separate and independent from the model training data during the measurement process. process.

\section{ACKNOWLEDGMENTS}

The research work is supported by grant VEGA 1/0393/18 „Research of Methods for Modeling and Compensation of Hysteresis in Pneumatic Artificial Muscles and PAM-actuated Mechanisms to Improve the Control Performance Using 
Computational Intelligence" and by EU Structural Funds project with ITMS code: 26220220103.

\section{REFERENCES}

[FESTO 2017] Fluidic muscle DMSP/MAS [online]. June 2017 [date of citing 2019-12-06]. https://www.festo.com/cat/sk_sk/data/doc_sk/PDF/SK/MAS_S K.PDF.

[HOSOVSKY 2016] Hosovsky, A. et al. Dynamic characterization and simulation of two-link soft robot arm with pneumatic muscles. Mechanism and Machine Theory, 2016, Vol. 103, pp. 98-116, ISSN 0094-114X.

[CHIBA 2014] Chiba, S. Dielectric Elastomers. Soft Actuators: Materials, Modeling, Applications, and Future Perspectives, Japan, Osaka: Springer, 2014, pp. 183-195, ISBN 978-4-43154766-2.

[KEEPING 2018] Keeping, S. What is a Collaborative Robot?. Collaborative robotics, Vol. 2, No. 1, pp. 7-8.

[KIKUCHI 2014] Kikuchi, K. and Tsuchitani, S. Ionic Conductive Polymers. Soft Actuators: Materials, Modeling, Applications, and Future Perspectives, Japan, Osaka: Springer, 2014, pp. 81-94, ISBN 978-4-431-54766-2.

[MISITI 2018] Misiti, A. A History of Industrial Robots. Collaborative robotics, Vol. 2, No. 1, pp. 9-10.
[OKUZAKI 2014] Okuzaki, H. Progress and Current Status of Materials and Properties of Soft Actuators. Soft Actuators: Materials, Modeling, Applications, and Future Perspectives, Japan, Osaka: Springer, 2014, pp. 3-18, ISBN 978-4-431-54766-2.

[SAMATHAM 2007] Samatham, R. et al. Active Polymers: An Overview. Electroactive Polymers for Robotic Applications, UK, London: Springer, 2007, pp. 1-36, ISBN 978-1-84628-372-7.

[TROJANOVA 2017] Trojanova, M. et al. Analysis of Dynamic Hysteresis of Manipulator Arm Driven by Fluid Muscles. Strojárstvo Extra 2017, No. 7-8, pp.92-93. ISSN 1335-2938.

[TROJANOVA 2018] Trojanova, M. et al. Comparison of Different Neural Networks Models for Identification of Manipulator Arm Driven by Fluidic Muscles. Acta Polytechnica Hungarica: An international peer-reviewed scientific journal of Óbuda University 2018, Vol. 15, No. 7, pp. 7-28, ISSN 2064-2687.

[TROJANOVA 2019] Trojanova, M. et al. Identification of planar arm dynamics with pneumatic artificial muscles using NARX MLP architecture. ARTEP 2019, Stara Lesna, Slovakia, February 6-8, 2019, Kosice: TUKE, 2019, pp. 05/1-05/14. ISBN 978-80-5533250-5.

[WICKRAMATUNGE 2010] Wickramatunge, K. C. et al. Study on mechanical behaviours of pneumatic artificial muscle. International Journal of Engineering Science 2010, Vol. 48, No. 2, pp. 188-198, ISSN 0020-7225.

\section{CONTACTS}

Ing. Monika Trojanova, PhD., Assoc. Prof. Ing. Alexander Hosovsky, PhD.

Technical University of Kosice, Faculty of Manufacturing Technologies with the Seat in Presov, Department of Industrial Engineering and Informatics, Bayerova 1, 08001 Presov, Slovakia +421 055602 6420, monika.trojanova@tuke.sk, alexander.hosovsky@tuke.sk, http://www.fvt.tuke.sk 